Reprod. Nutr. Dévelop., 1980, 20 (4 B), 1155-1159.

\title{
Rôle des afférences splanchniques de la zone duodéno-pancréatique dans le vomissement
}

\author{
par L. GARNIER, J. P. ALBANO \\ Laboratoire de Neurophysiologie viscérale, Université de Provence, \\ Centre St-Jérôme, 13397 Marseille Cedex 4, France
}

Summary. The role of splanchnic afferences of the duodeno-pancreatic zone in vomiting.

The duodenum and pancreas of the cat send splanchnic afferences via the anterio $r$ nerve branches. The latter run along the cœliac artery.

The stimulation of those pathways induced pattern alteration of the respiratory command signal. After an interruption of phrenic nerve discharge, the respiratory function was mobilized to empty the stomach. It is suggested that the duodenum is systematically involved in all types of vomiting.

\section{Introduction.}

Dans le cadre d'un travail sur les effets respiratoires de la stimulation des afférences splanchniques chez le Chat légèrement anesthésié (Albano et Garnier, 1977), notre attention a été incidemment relenue par les efforts de vomissement de certains animaux, lorsque la zone duodéno-pancréatique était stimulée soit mécaniquement, soit électriquement. Nous avons entrepris une étude systématique, en nous proposant de rechercher les mécanismes sous-jacents au phénomène observé. La littérature qui soutient des rapports directs ou indirects avec notre problème ne comporte qu'un nombre réduit de travaux parmi lesquels on relèvera ceux de Schrager et lvy (1928), Morin ef Vial (1934), Borison et Wang (1949), Lumsden et Holden (1969), Weisbrodt et Christensen (1972), Monges, Salducci et Naudy (1973). Dans leurs études classiques, Borison et Wang $(1949,1953)$ soulignent les difficultés de l'approche expérimentale du problème dont la solution paraît être enfermée dans un cercle vicieux : si l'animal esł anesthésié, le vomissement ne peut avoir lieu ; si l'animal est éveillé, les efforts de vomissement déplacent les systèmes de recueil des paramètres étudiés. En utilisant des animaux très légèrement anesthésiés, nous avons presque toujours enregistré les prodromes respiratoires du vomissement mais l'acte lui-même ne survient que dans une proportion relativement réduite des cas : environ un tiers. 


\section{Matériel et méthodes.}

L'animal utilisé est le Chat anesthésié au moyen de Nesdonal (Penthiobarbital) injecté à dose faible par la voie intrapéritonéale $(30 \mathrm{mg} / \mathrm{kg})$. Sur une trentaine d'animaux pesant entre 2,3 et $3,5 \mathrm{~kg}$, on pratique du côté gauche une incision abdominale parallèle aux côtés, permettant de dégager le duodénum, le pancréas, l'estomac, le ganglion coeliaque et éventuellement le nerf grand splanchnique dans sa portion sous-diaphragmatique. L'activité respiratoire est enregistrée au niveau du nerf phrénique isolé dans son parcours cervical. Dans certaines expériences on enregistre simultanément l'évolution de la pression intra-thoracique au moyen d'un cathéter inséré d'une manière étanche dans le sixième espace intercostal. La stimulation électrique est appliquée sur le duodénum ou le pancréas au moyen de deux fils de cuivre émaillé, séparés d'environ $5 \mathrm{~mm}$ et implantés tangentiellement à l'organe. Un générateur d'impulsions délivre des chocs de durée, d'intensité et de fréquence déterminées. La stimulation mécanique par distension du duodénum est opérée par le moyen d'un ballonnet plus ou moins gonflé d'air. La pression artérielle recueillie au niveau de l'artère fémorale et la pression intra-thoracique sont mesurées au moyen de capteurs de type capacitif. L'ensemble des phénomènes est enregistré sur un inscripteur à jet d'encre (Mingograf).

\section{Résultats.}

1) La distension modérée du duodénum au moyen de 20 à $30 \mathrm{ml}$ d'air (fig. 1) est accompagnée d'une perturbation respiratoire caractérisée par une apnée de durée variable, suivie d'un changement de type de la respiration qui se caractérise par une précipitation et une amplification des volées du nerf phrénique. Cette forme de perturbation spécifique est rencontrée chaque fois que, sur un animal convenablement anesthésié, on distend le duodénum ou on stimule électriquement le corps du pancréas, ou les fibres afférentes qui longent l'artère cœliaque, ou la portion centrale du nerf splanchnique ligaturé. Le phénomène persiste après bivagotomie. Il est moins marqué après la section du nerf splanchnique droit. En général, la perfurbation respiratoire engendrée se dissipe assez rapidement après la fin des stimulations.



FIG. 1. - Perturbations respiratoires induites par distension duodénole. Tracé supérieur : nerf phrénique; Tracé moyen : pression artérielle ; Tracé inférieur : gonflement du ballonnet intraduodénal.

2) Dans certains cas favorables, le changement de mode respiratoire précédemment noté se maintient pendant une durée plus ou moins longue et se prolonge par des efforts de vomissement ef par l'acte de vomissement lui-même (fig. 2). En 
dehors d'un artéfact de translation de l'enregistrement de la pression artérielle (A3) dô à des imperfections techniques, on constate en $A$ et $B$ les faits suivants :

A. Les deux groupes de tracés sont en continuité. - La distension modérée du duodénum (ligne 4) réduit l'amplitude et la durée des décharges phréniques (ligne 1) ainsi que celles des variations de la pression intrathoracique (ligne 2). Après la distension, les décharges phréniques s'amplifient et s'accompagnent de décharges erratiques (indiquées au moyen de flèches) que l'on peut également lire sur le tracé de la pression intrathoracique sous forme d'encoches concomitantes. 50 secondes après le débuł de la distension, les décharges phréniques s'amplifient considérablement et se
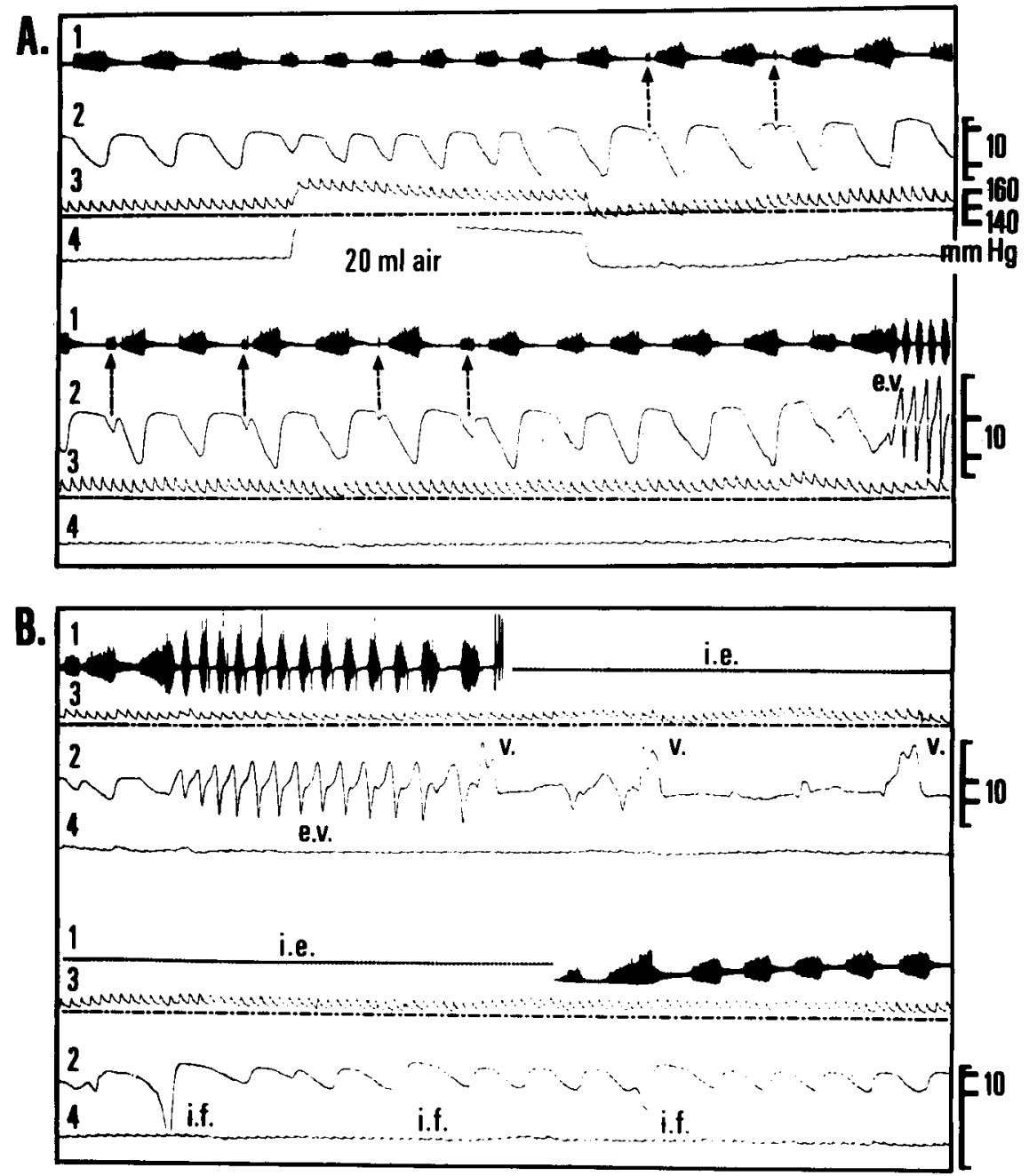

1: N.Phr. , 2:P. intrathorac. , 3 : P. art. , 4 : Dist.Duodén. 1s

FIG. 2. - Déclenchement du réflexe de vomissement par distension du duodénum. 1 : nerf phrénique ; 2 : pression intrathoracique ; 3 : pression artérielle ; 4 : gonflement du ballonnet intraduodénal. 
précipitent. L'animal est secoué d'efforts de vomissement (e.v.). Tout au long de l'enregistrement, la pression artérielle reste relativement stable (ligne 3).

B. Les deux groupes de tracés sont en continuité. - Sur le même animal, il a été possible d'enregistrer une autre séquence d'efforts de vomissement. Lors de l'expulsion du contenu gastrique (e.v.), l'enregistrement des décharges phréniques est interrompu par impossibilité technique (i.e.). On peut cependant suivre l'évolution de la respiration grâce au tracé de la pression intrathoracique. On constate de longues inspirations coupées de brèves et puissantes expirations. Après les phases de vomissement, des inspirations forcées (i.f.) précèdent la restauration de l'état de repos; il est alors possible d'enregistrer de nouveau l'activité du nerf phrénique.

\section{Discussion.}

Dans un précédent travail (Albano et Garnier, 1977), nous avons identifié, au sein des afférences splanchniques, un certain nombre de fibres contenues dans les rameaux duodéno-pancréatiques dont la stimulation reproduif les perturbations respiratoires qui précèdent le vomissement. Ces rameaux comportent un nombre sensiblement égal de fibres amyéliniques et myéliniques. Parmi ces dernières, celles dont le diamètre est compris entre 3 et $5 \mu \mathrm{m}$, au nombre de $207 \pm 10(\mathrm{~N}=6)$, sont majoritaires. Elles pourraient constituer le support hodologique des phénomènes que nous observons et représentent, peut-être, une voie afférente privilégiée des messages qui, au niveau des structures voisines de l'obex, coordonnent les réactions comportementales respiratoires et circulatoires contemporaines du vomissement. Ces faits paraissent en accord avec les résultats obtenus par Ingelfinger et Moss (1942) après stimulation des canaux semi-circulaires ou après injection sous-cutanée de morphine et illustrés par le déclenchement simultané d'un spasme duodénal accompagné de sensation de nausée. Cette antériorité du spasme duodénal sur tous les autres phénomènes qui peuvent aboutir au vomissement est également soulignée dans les travaux de Luckhardt, Philipps et Carlson (1919), Keeton (1925), Wolf et Wolff (1947).

En ce qui concerne les « centres bulbaires» du réflexe tels que les délimitent Borison ef Wang (1949), ils coïncident ou se superposent avec les «centres respiratoires » eux-mêmes ou la formation réticulaire voisine. Cefte coïncidence topographique des «centres» pourrait expliquer l'intervention précoce des perturbations respiratoires dans l'acte de vomissement ef le rôle fondamental des muscles respiratoires dans les mécanismes d'expulsion du contenu gastrique.

\section{Conclusion.}

Un contingent particulier d'afférences splanchniques paraît être au service de la protection d'une zone stratégique essentielle des phénomènes digestifs. Dans les situations critiques, des récepteurs de la région duodéno-pancréatique déclenchent les signaux d'alerte qui conduisent par voie réflexe à l'expulsion du contenu gastroduodénal. Dans ce cas, l'antagonisme vago-sympathique est illustré par une oppo- 
sition de finalité fonctionnelle : tandis que les afférences vagales sont au service de réflexes régulateurs, les afférences splanchniques assument des fonctions de protection du tube digestif.

\begin{abstract}
Journées Ingestion-Digestion-Absorption, de l'Association française de Nutrition, Paris, 15-16 novembre 1979.
\end{abstract}

\title{
Références
}

ALBANO J. P., GARNIER L., 1977. Effets respiratoires de la stimulation des afférences splanchniques chez le Chat légèrement anesthésié. Arch. ital. Biol., 115, 1-19.

BORISON H. L., WANG S. C., 1949. Functional organization of central coordinating mechanism for emesis in cat. J. Neurophysiol., 12, 305-313.

BORISON H. L., WANG S. C., 1953. Physiology and pharmacology of vomiting. Pharmacol. Rev., 5, 193-230.

INGELFINGER F. J., MOSS R. E., 1942. The activity of the descending duodenum during nausea. Am. J. Physiol., 136, 561-566.

KEETON R. W., 1925. Nausea and related sensations elicited by duodenal stimulation. Arch. int. Med., 35, 687-697.

LUCKHARDT A. B., PHILIPPS H. T., CARLSON A. J., 1919. Contribution to the physiology of the stomach. Am. J. Physiol., 50, 57-66.

LUMSDEN K., HOLDEN W. S., 1969. The act of vomiting in man. Gut, 10, 173-179.

MONGES H., SALDUCCI J., NAUDY B., 1973. Electrical activity of the gastro-intestinal fract in dog during vomiting. Proc. 4th int. Symp. Gostro-intestinal motility, Banff, Alberta, Canada. Mitchell Press, Vancouver, 479-488.

MORIN G., VIAL J., 1934. Réactions respiratoires et circulatoires provoquées par distension brusque de l'intestin chez le chien. Arch. int. Physiol., 38, 428-442.

SCHRAGER V. L., IVY A. C., 1928. Symptoms produced by distension of the gallbladder and biliary ducts. Surg. Gynec. Obst., 47, 1-13.

WEISBRODT N. W., CHRISTENSEN J., 1972. Electrical activity of the cat duodenum in fasting and vomiting. Gastroenterology, 63, 1004-1010.

WOLF S., WOLFF H. G., 1947. Human gastric function, on experimental study of a man and his stomach. Oxford Univ. Press, New York City, 262 pp. 